\title{
Involvement of p62 Nucleoporin in Angiotensin II-Induced Nuclear Translocation of STAT3 in Brain Neurons
}

\author{
Di Lu, Hong Yang, and Mohan K. Raizada \\ Department of Physiology, College of Medicine, University of Florida, Gainesville, Florida 32610
}

Chronic stimulation of brain neurons by angiotensin II (Ang II) results in a increase in norepinephrine (NE) uptake. This involves stimulation of transcription of NE transporter and tyrosine hydroxylase genes and is associated with translocation of signaling molecules and transcription factors from the cytoplasmic compartment into the neuronal nucleus ( $\mathrm{Lu}$ et al., 1996a). We report here that the phosphorylation of p62, a glycoprotein nucleoporin of the nuclear pore complex (NPC), by MAP kinase is involved in this process. Ang II caused a timedependent translocation of signal transducers and activators of transcription (STAT3) from the cytoplasmic compartment into the nucleus. This translocation was attenuated by pretreatment with antisense oligonucleotide (AON) to MAP kinase. Ang II also stimulated phosphorylation of p62, and a maximal phosphorylation of 12-fold was observed with 100 nm Ang II. This stimulation was blocked by losartan, an $\mathrm{AT}_{1}$ receptor subtypespecific antagonist. The conclusion that MAP kinase is involved in Ang II-induced phosphorylation of p62 and nuclear translo- cation of STAT3 is supported by the following. (1) p62 phosphorylation was blocked by a peptide that competes with p62 as a MAP kinase substrate both in vitro and in vivo; (2) AON to MAP kinase attenuated Ang II stimulation of p62 phosphorylation; and (3) in addition, it also blocked nuclear translocation of STAT3. Intracellular loading of the peptide containing MAP kinase substrate consensus of the p62 reduced Ang II stimulation of p62 phosphorylation and nuclear translocation of STAT3 in both in vivo and in vitro experiments. These observations suggest that Ang II-induced phosphorylation of p62 may accelerate the activity of the NPC, which would result in an increase in the nuclear transport of transcription factors and signaling molecules. This will stimulate transcriptional processes associated with Ang II regulation of NE neuromodulation.

Key words: angiotensin; MAP kinase; nuclear translocation; signal transduction; p62; nuclear pore complex
Ang II exerts chronic norepinephrine (NE) neuromodulatory actions by stimulating the expression of genes for norepinephrine transporter (Lu et al., 1996b), tyrosine hydroxylase and dopamine $\beta$-hydroxylase (Yu et al., 1996), key components in the regulation of turnover, synthesis, and release of catecholamines. Recent studies have indicated that the interaction of Ang II with the $\mathrm{AT}_{1}$ receptor subtype, a member of the G-protein-coupled receptor superfamily, initiates a cascade of signaling events involving RasRaf-MAP kinase and Jak/signal transducers and activators of transcription (STAT) pathways. Stimulation of these signaling molecules and transcription factors such as Fos, Jun, and STAT mediates in their translocation into the nuclear compartment that results in the chronic actions of this hormone (Marrero et al., 1995; Lu et al., 1996a; Yang et al., 1996). Despite identification of these signaling molecules, little is known about the regulatory mechanism by which they are transported across the nuclear membrane to mediate the chronic effects of Ang II. In fact, there is paucity of information regarding the mechanism of translocation of macromolecules across the nuclear membrane in general (Akey, 1992; Davis, 1995; Melchior and Gerace, 1995).

Received Sept. 12, 1997; revised Dec. 4, 1997; accepted Dec. 5, 1997.

The research was supported by National Institutes of Health Grant HL-32610. We thank Dr. John Hanover of National Institutes of Health for providing the p62 antibody and Dr. Carl Feldherr for rhodamine-labeled BSA-NLS fusion protein and its mutant counterpart. In addition, we also acknowledge Dr. Feldherr's valuable suggestions throughout this work and his critical review of this manuscript.

Correspondence should be addressed to Dr. Mohan K. Raizada, Department of Physiology, College of Medicine, University of Florida, P.O. Box 100274, Gainesville, FL 32610.

Copyright (C) 1998 Society for Neuroscience $\quad 0270-6474 / 98 / 181329-08 \$ 05.00 / 0$
The transport of large molecules between the cytoplasm and the nucleus is a key regulatory event in the transmission of chemical signals that are generated by the activation of cell surface receptors. This transport is facilitated by the nuclear pore complex (NPC). The NPC is a large macromolecular assembly consisting of an intricate arrangement of proteins and cofactors that perforate the nuclear envelope (Akey, 1992; Davis, 1995). Transport takes place along the pore axis and involves an initial docking step in which an interaction with the NPC proteins is important (Newmeyer and Fordes, 1988; Richardson et al., 1988; Moore and Blobel, 1992; Boulikas, 1993; Melchior and Gerace, 1995). This is followed by the translocation through the pore in an energy-dependent process (Newmeyer and Fordes, 1988; Richardson et al., 1988; Moore and Blobel, 1992, 1993; Melchior et al., 1993). In recent years considerable progress has been made in characterizing many protein components of the NPC (Gerber and Gerace, 1992; Shi and Thomas, 1992; Sukegawa and Blobel, 1993; Miller and Hanover, 1994; Moore and Blobel, 1994). This has led to the identification of a series of proteins, collectively called nucleoporins. The p62 nucleoporin is of particular interest for its involvement in nuclear transport (Starr and Hanover, 1990; Finlay et al., 1991; Panté and Aebi, 1994). It is a glycoprotein that contains a large number of O-linked $N$-acetylglucosamine residues and has been demonstrated to be directly involved in the nuclear transport process (Starr and Hanover, 1990; Finlay et al., 1991; Miller and Hanover, 1994; Panté and Aebi, 1994). Many studies on the structural-functional aspects of this protein have led to the hypothesis that p62 could be phosphorylated, and phosphorylation and dephosphorylation of p62 may be a crucial 
step in the energy-dependent translocation of macromolecules through the NPC (Finlay et al., 1991; Kita et al., 1993; Buss and Stewart, 1995). This study was designed to investigate the phosphorylation of p62 and its role in nuclear translocation. It shows that Ang II stimulates MAP kinase-dependent phosphorylation of p62 and that Ang II-stimulated translocation of STAT3 into the nucleus is dependent on the phosphorylation of p62.

\section{MATERIALS AND METHODS}

One-day-old Wistar Kyoto strain of rats were obtained from our breeding colony, which originated from Harlan Sprague Dawley (Indianapolis, IN). DMEM, plasma-derived horse serum (PDHS), and $1 \times$ crystalline trypsin $(150 \mathrm{U} / \mathrm{mg})$ were obtained from Central Biomedia (Irwin, MO). Ang II was purchased from Sigma (St. Louis, MO). Losartan potassium was a gift from DuPont Merck (Wilmington, DE), and PD123319 was from RBI (Natick, MA). [ $\left.{ }^{32} \mathrm{P}\right]$ orthophosphate $(1 \mathrm{mCi}=37 \mathrm{MBq})$, $\left[\gamma_{-}{ }^{32} \mathrm{P}\right]$-ATP $(3000 \mathrm{Ci} / \mathrm{mmol})$, and chemiluminescence assay kit were from DuPont NEN (Boston, MA). Monoclonal anti-STAT3, antiphosphotyrosine antibodies, horseradish-peroxidase-conjugated antimouse antibody, and protein $\mathrm{A}+\mathrm{G}$ conjugated-agarose beads were purchased from Santa Cruz Biotechnology (Santa Cruz, CA). Monoclonal anti-NPC414 antibody was purchased from BabCo (Richmond, CA), and monoclonal anti-p62 specific antibody was a gift from Dr. John Hanover (National Institute of Diabetes and Digestive and Kidney Diseases, National Institutes of Health, Bethesda, MD). FITC-conjugated antimouse antibody was from Boehringer Mannheim Biochemicals (Indianapolis, IN). BSA-nuclear localization signal (NLS) fusion protein and its control counterpart were gifts from Dr. Carl Feldherr (Department of Anatomy and Cell Biology, University of Florida, Gainesville, FL). BSA-NLS contained NLS of simian virus 40 large T antigen conjugated with rhodamine-labeled BSA. Control fusion protein was the same as BSA-NLS except NLS sequence was omitted. Oligonucleotide sense (SON) and antisense (AON) to MAP kinase were synthesized in the DNA Synthesis Facility of the Interdisciplinary Center for Biotechnology Research (University of Florida), essentially as described previously (Yang et al., 1996). Synthetic peptide corresponding to the p62 amino acid sequence of 189 to 198 (GSPFTPATLA) and its mutant where the $\mathrm{Thr}^{193}$ was substituted with Ala were synthesized by Genemed Biotechnologies (San Francisco, CA). All other biochemicals were from Fisher Scientific (Pittsburgh, PA) and were of the highest grade available.

\section{Preparation of neuronal cultures from WKY rat brains}

Hypothalamus-brainstem areas of 1-d-old rat brains were dissected, and brain cells were dissociated by trypsin. The hypothalamic block contained the paraventricular nucleus, the supraoptic, anterior, lateral, posterior, dorsomedial, and ventromedial nuclei, whereas the brainstem block contained the medulla oblongata and pons. Trypsin-dissociated brain cells were plated onto poly-L-lysine precoated $100 \mathrm{~mm}$ tissue culture dishes $\left(2 \times 10^{7}\right.$ cells/dish $)$ in DMEM containing $10 \%$ PDHS, and the neuronal cultures were established essentially as described previously (Sumners et al., 1991; Raizada et al., 1993). Immunohistochemical analysis with the use of neuron-specific antigens has shown that these cultures contain at least $90 \%$ neuronal cells (Raizada et al., 1994). The remaining cells were of the astroglial type (Raizada et al., 1994). Neurons established in culture for $15 \mathrm{~d}$ were used throughout this study.

\section{Measurement of STAT3 phosphorylation in cytoplasmic and nuclear fractions}

Isolation of cytoplasmic and nuclear fractions of neurons. Fractionation of neuronal cells to separate cytoplasmic and nuclear fractions was performed essentially as described elsewhere (Yang et al., 1997). This protocol is based on the method of Newmeyer et al. (1986). In brief, neuronal cells were rinsed twice with PBS, $\mathrm{pH} 7.4$, and cells were scraped off the dish; the resulting cell pellet was suspended in $10 \mathrm{~mm}$ HEPES, $\mathrm{pH}$ 7.4, containing $10 \mathrm{~mm}$ potassium acetate, $1.5 \mathrm{~mm}$ magnesium chloride, 0.5 $\mathrm{mM}$ DTT, $0.2 \mathrm{~mm}$ PMSF, and $1 \mu \mathrm{g} / \mathrm{ml}$ each aprotinin, leupeptin, and pepstatin for $15 \mathrm{~min}$ on ice. The cells were homogenized with 15 gentle strokes using a type-B pestle in a Dounce homogenizer. Completion of homogenization was ascertained by microscopic examination. The cytoplasmic extract was collected by centrif ugation at $3300 \times g$ for $15 \mathrm{~min}$ and dialyzed against transport buffer (20 mM HEPES, pH 7.3, $110 \mathrm{~mm}$ potassium acetate, $5 \mathrm{~mm}$ sodium acetate, $1.5 \mathrm{~mm}$ magnesium chloride, 1 mM EGTA, $0.5 \mathrm{~mm}$ DTT, and $1 \mu \mathrm{g} / \mathrm{ml}$ each aprotinin, leupeptin, and pepstatin) overnight at $4^{\circ} \mathrm{C}$. This fraction was used for STAT3 immunoprecipitation experiments as described below. The pellet containing nuclei was lysed by nuclear lysis buffer ( $25 \mathrm{~mm}$ Tris-Cl, $\mathrm{pH} 7.4,25 \mathrm{~mm}$ $\mathrm{NaCl}, 1 \%$ Triton $\mathrm{X}-100,1 \%$ deoxycholic acid, $1 \mathrm{~mm}$ sodium orthovanadate, $1 \mathrm{~mm}$ phenylmethylsulfonyl fluoride, $10 \mu \mathrm{g} / \mathrm{ml}$ aprotinin, and 0.8 $\mu \mathrm{g} / \mathrm{ml}$ leupeptin), and centrifuged at $12,000 \times \mathrm{g}$ for $5 \mathrm{~min}$ at $4^{\circ} \mathrm{C}$. The supernatant was saved as nuclear extract. The purity of both the cytoplasmic and nuclear fractions was ascertained with the use of standard markers (Jakob, 1992). The nuclear fraction was found to be free of the cytoplasmic marker aldolase. The contamination of cytoplasmic fraction by nuclear markers, as measured by the levels of lemnin, was only $10 \%$. Measurement of STAT3 phosphorylation. Cytoplasmic and nuclear fractions (200 $\mu \mathrm{g}$ protein) were used for immunoprecipitation with the use of anti-phosphotyrosine antibody overnight at $4^{\circ} \mathrm{C}$. Immune complexes were collected on agarose beads conjugated with protein $A+G$ and after they were washed three times with the wash buffer $(50 \mathrm{~mm}$ Tris- $\mathrm{Cl}, \mathrm{pH}$ 7.4, $20 \mathrm{mM} \mathrm{MgCl}_{2}$, and $150 \mathrm{~mm} \mathrm{NaCl}$ ), they were suspended in Laemmli's buffer, boiled for $3 \mathrm{~min}$, and subjected to SDS-PAGE (Yang et al., 1996). Samples were transferred to PVDF membrane and membranes used for immunoblotting with anti-STAT3 monoclonal antibody as described previously (Yang et al., 1996).

\section{Immunocytochemical staining of STAT3}

The protocol used was essentially as described by us previously except that STAT3 antibody was used and cells were examined by confocal microscopy (Lu et al., 1996a,b; Yang et al., 1996). Approximately 200300 cells were examined, and results are representative of such examination.

\section{$M A P$ kinase $A O N$ and SON treatments}

AON and SON corresponding to a region for both p44 and p42 cDNAs of the MAP kinase were synthesized (Yang et al., 1996). The oligonucleotides were made as phosphorothiocate derivatives to enhance nuclease resistance. Neuronal cultures established for $15 \mathrm{~d}$ were treated with $1 \mu \mathrm{M}$ AON or SON to MAP kinase dissolved in $2 \mu \mathrm{g} / \mathrm{ml}$ Lipofectin Reagents (Life Technologies) for $24 \mathrm{hr}$ at $37^{\circ} \mathrm{C}$, before the experiments.

\section{Measurement of p62 phosphorylation}

Neuronal cells were rinsed in phosphate-free DMEM and incubated with $2 \mathrm{mCi}\left[{ }^{32} \mathrm{P}\right]$-orthophosphate in phosphate-free DMEM for $2 \mathrm{hr}$ at $37^{\circ} \mathrm{C}$ to label the intracellular ATP pools. This was followed by incubation with or without $100 \mathrm{~nm}$ Ang II at $37^{\circ} \mathrm{C}$. The cells were rinsed six times with ice-cold PBS, pH 7.4, followed by lysis for $20 \mathrm{~min}$ on ice in lysis buffer (25 mm Tris-HCl, pH 7.4, 25 mm NaCl, 1\% Triton X-100, 1\% deoxycholic acid, $0.1 \%$ SDS, $1 \mathrm{~mm}$ sodium orthovanadate, $10 \mathrm{~mm}$ sodium fluoride, $10 \mathrm{~mm}$ sodium pyrophosphate, $0.5 \mathrm{~mm}$ EGTA, $1 \mathrm{~mm}$ phenylmethylsulfonyl fluoride, $10 \mu \mathrm{g} / \mathrm{ml}$ aprotinin, and $0.8 \mu \mathrm{g} / \mathrm{ml}$ leupeptin). Samples were centrifuged at $12,000 \times g$ for $5 \mathrm{~min}$ at $4^{\circ} \mathrm{C}$, and supernatants containing $200 \mu \mathrm{g}$ of protein were used for immunoprecipitation with either anti-NPC414 $(5 \mu \mathrm{g})$ or anti-p62 (5 $\mu \mathrm{g})$ specific antibody overnight at $4^{\circ} \mathrm{C}$. Immune complexes were collected on agarose beads conjugated with protein $A+G$, suspended in Laemmli's sample buffer, boiled for $3 \mathrm{~min}$, and subjected to SDS-PAGE followed by autoradiography. The radiolabeled band corresponding to a $62 \mathrm{kDa}$ protein was quantitated essentially as described previously (Lu et al., 1996a,b; Yang et al., 1996).

\section{Phosphorylation of p62 by exogenous MAP kinase}

Immunoprecipitates of p62 from neuronal cells were obtained using a protocol described above except the $\left[{ }^{32} \mathrm{P}\right]$-orthophosphate step was excluded. The immune complex was subject to exogenous MAP kinase assay by a protocol based on Paxon et al. (1994). Briefly, the pellet containing p62 immunoprecipitate was washed twice and suspended in 20 $\mu l$ MAP kinase assay buffer (50 mM HEPES, pH 7.5, $0.1 \mathrm{~mm}$ EDTA, $0.015 \%$ Triton X-100). This was followed by mixing with $20 \mu \mathrm{l}$ of MAP kinase solution ( $0.3 \mathrm{U}$ MAP kinase containing $0.1 \mathrm{mg} \mathrm{BSA} / \mathrm{ml}$ and $0.2 \%$ $\beta$-mercaptoethanol) and $20 \mu \mathrm{l}$ of ATP mixture $(0.3 \mathrm{~mm}$ ATP, $30 \mathrm{~mm}$ $\mathrm{MgCl}$, and $200 \mu \mathrm{Ci}\left[\gamma_{-}{ }^{32} \mathrm{P}\right]-\mathrm{ATP} / \mathrm{ml}$ in kinase assay buffer) to start the reaction. The reaction was stopped by adding $15 \mu \mathrm{l}$ of $5 \times$ Laemmli's sample buffer at indicated time periods and boiling for $3 \mathrm{~min}$. The sample was then subjected to SDS-PAGE electrophoresis and autoradiography.

\section{Osmotic loading of p62 peptide ${ }^{189-198}$}

Osmotic loading of p62 peptide ${ }^{189-198}$ was performed essentially by the method of Ahmad et al. (1995). In brief, neuronal cells were rinsed with 
PBS, pH 7.4, and incubated for 10 min with a loading solution $[0.5 \mathrm{M}$ sucrose, $10 \%$ polyethylene glycol $1000,10 \%$ fetal bovine serum, and 200 $\mu \mathrm{g} / \mathrm{ml}$ p62 peptide ${ }^{189-198}$ or its mutant peptide ( $\mathrm{Thr}^{193}$ replaced by Ala) in DMEM buffered with $25 \mathrm{~mm}$ HEPES, $\mathrm{pH}$ 6.8]. This was followed by a rapid rinse with a hypotonic solution $\left(6.5\right.$ vol $\mathrm{H}_{2} \mathrm{O}: 3.5$ vol DMEM, buffered with $25 \mathrm{~mm}$ HEPES, pH 6.8), essentially as described elsewhere (Ahmad et al., 1995). After this treatment, cells were incubated with DMEM containing 10\% PDHS and were subjected to $\left[{ }^{32} \mathrm{P}\right]-$ orthophosphate labeling and p62 phosphorylation analysis as described above.

\section{Determination of the effect of peptide ${ }^{189-198}$ on Ang II- stimulated STAT3 accumulation in isolated nuclei}

Neuronal cells were subjected to osmotic loading of p62 peptide ${ }^{189-198}$ or its mutant form with appropriate controls. The controls included cells subjected to osmotic shock without peptide, or with bovine serum albumin or a structurally unrelated peptide corresponding to the $\mathrm{AT}_{1}$ receptor sequence (amino acids 295 to 315). These controls acted like mutant peptide and showed no effect. This was followed by stimulation of cells with $100 \mathrm{~nm}$ Ang II for $10 \mathrm{~min}$ at $37^{\circ} \mathrm{C}$. Cells were collected, lysed, and centrifuged at $500 \times g$ for $10 \mathrm{~min}$. The resulting cytoplasmic and nuclear fraction were collected (Yang et al., 1997) and used for in vitro transport assay. The assay was essentially based on a protocol of Feldherr (1995). In brief, nuclei $\left(3 \times 10^{4}\right)$ from cells after various treatments were suspended in a transport buffer containing ATP and combined with the cytoplasmic fractions containing $\sim 500 \mu \mathrm{g}$ of protein. After incubation for $15 \mathrm{~min}$ at $30^{\circ} \mathrm{C}$, the nuclei were washed once with the transport buffer, plated on the glass slides, and subjected to STAT3 immunocytochemistry followed by confocal microscopic analysis (Lu et al., 1996a,b). In initial recombination experiments we had established that the nuclear translocation of STAT3 is ATP sensitive and thus constitutes a specific nuclear transport process in this preparation. This observation is consistent with other systems (Feldherr, 1995; Radu et al., 1995a).

\section{Experimental groups and data analysis}

Each data point in all experiments was obtained from three culture dishes, and each experiment was performed at least three times. Data presented are mean \pm SE. Comparisons between data points were made by using one-way ANOVA and Dunnett's test from the Statistician software. All immunofluorescent experiments were repeated at least six times, and 200-300 cells were examined in each staining and representative images were captured. Bands on autoradiograms were quantitated with the use of an SW5000 Gel Analysis System (Ultra Violet Products), as described previously (Lu et al., 1996b; Yang et al., 1996). The linearity of the absorbance for each band was established in initial experiments by controlling the exposure times of immunoblot with the x-ray film. The absorbance of each band was normalized for an equal amount of protein loading.

\section{RESULTS}

\section{Effects of Ang II on neuronal STAT3}

Previously it has been shown that Ang II stimulates phosphorylation of STAT3 in vascular smooth muscle cells (Marrero et al., 1995). In this study, we first determined whether a similar effect of Ang II on STAT3 is also seen in the neurons. Incubation of neuronal cells with Ang II resulted in a time-dependent stimulation of STAT3 phosphorylation. A maximal, 12-fold stimulation of phosphorylation was observed in $30 \mathrm{~min}$ by $100 \mathrm{~nm}$ Ang II. The stimulation was mediated by the interaction of Ang II with the $\mathrm{AT}_{1}$ receptor subtype because it was blocked by losartan, its antagonist, and not by PD123319, an $\mathrm{AT}_{2}$ receptor subtype antagonist. Figure 1 shows that Ang II stimulated translocation of phosphorylated STAT3 into the nucleus. Initially, levels of phosphorylated STAT3 were increased in the cytoplasm, followed by its increase in the nuclear fraction. The nuclear levels reached optimum in $15 \mathrm{~min}$, and by $30 \mathrm{~min}$ the levels in the nucleus reached a plateau and were fivefold higher than in the cytoplasm. Confirmation of a nuclear translocation of STAT3 was provided by immunofluorescence data (Fig. 2). STAT3 was primarily localized in the cytoplasmic compartment in control neurons (Fig.

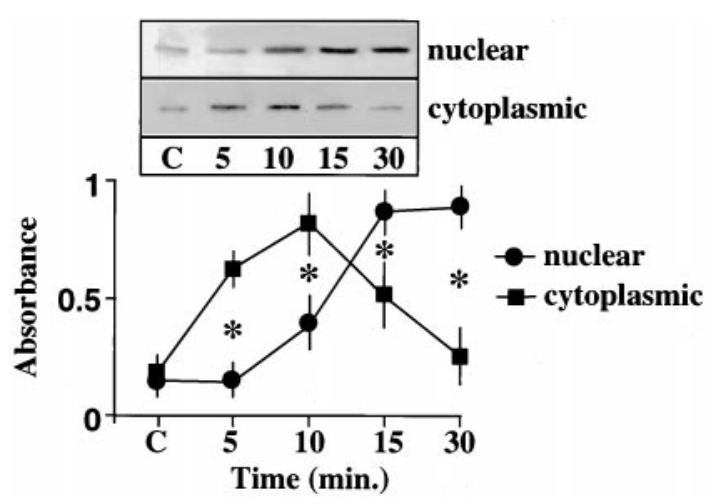

Figure 1. Effect of Ang II on STAT3 phosphorylation and its translocation into the nucleus. Neurons were incubated with $100 \mathrm{~nm}$ Ang II for the indicated time period, and cells were used to isolate cytoplasmic and nuclear fractions as described in Materials and Methods. The fractions were subjected to immunoprecipitation with anti-phosphotyrosine antibody followed by immunoblotting with the use of specific antibody to STAT3 essentially as described in Materials and Methods. Top, A representative autoradiogram. Bottom, Bands corresponding to STAT3 were quantitated with the use of the SW $5000 \mathrm{Gel}$ Analysis System. Data were normalized for equal protein loading and presented as mean $\pm \mathrm{SE}(n=$ 3 ), as described previously (Yang et al., 1996). *Significantly different; $p<$ 0.05 ; nuclear versus cytoplasmic.
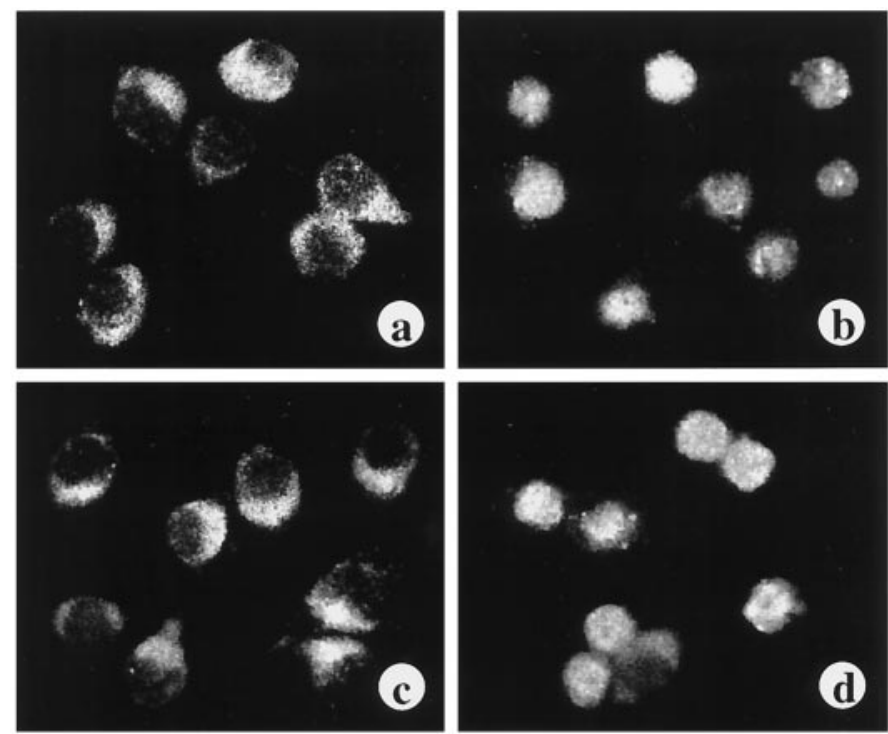

$10 \mu$

Figure 2. Immunofluorescent distribution of STAT3 in Ang II-treated neurons. Experimental conditions were essentially as described in the legend to Figure 1. $a$, Control neurons depicting predominant staining of STAT3 immunoreactivity in the cytoplasmic compartment. $b$, Treatment of neurons for 30 min with $100 \mathrm{~nm}$ Ang II results in STAT3 redistribution into the nuclear compartment. $c$, Treatment of neurons with $10 \mu \mathrm{M}$ losartan, an $\mathrm{AT}_{1}$ receptor subtype specific antagonist, completely blocks the Ang II-induced nuclear translocation of STAT3. $d$, Lack of effect of Ang II-induced nuclear translocation by incubation with $10 \mu \mathrm{M}$ $\mathrm{PD} 123319$, an $\mathrm{AT}_{2}$ receptor subtype specific antagonist.

$2 a$ ). After incubation with $100 \mathrm{~nm}$ Ang II for 30 min that results in its phosphorylation, STAT3 localization was shifted from the cytoplasm to the nucleus (Fig. 2b). This translocation was blocked by $10 \mu \mathrm{M}$ losartan (Fig. 2c) and not by $10 \mu \mathrm{M}$ PD123319 (Fig. $2 d$ ).

Next, we determined whether MAP kinase is involved in the 
A
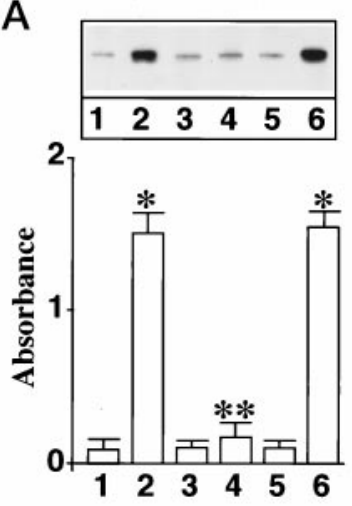

B
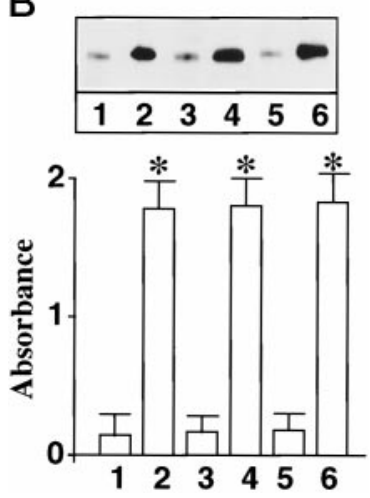

Figure 3. Effect of MAP kinase antisense oligonucleotide on Ang IIinduced translocation of STAT3 into the nuclear fraction. $A$, Neuronal cultures were incubated without $(1,2)$ or with $1 \mu \mathrm{M}$ MAP kinase AON $(3$, 4 ) or with $1 \mu \mathrm{M}$ MAP kinase $\operatorname{SON}(5,6)$ for $24 \mathrm{hr}$ at $37^{\circ} \mathrm{C}$. In parallel experiments this treatment causes a $70-80 \%$ decrease in MAP kinase immunoreactivity. After this, neurons were incubated without $(1,3,5)$ or with $100 \mathrm{~nm}$ Ang II $(2,4,6)$ for $30 \mathrm{~min}$. Nuclear fractions were isolated and levels of phosphorylated STAT3 were quantitated essentially as described in Materials and Methods. Top, A representative autoradiogram. Bottom, Data from three separate experiments as mean \pm SE. *Significantly different from $1 ; p<0.005$. **Significantly different from 2 ; $p<0.005$. $B$, The experimental protocol was identical to the one described in $A$, except that the effect of Ang II on levels of STAT3 phosphorylation in whole-cell lysates was analyzed. Data are mean $\pm \mathrm{SE}$; $n=3$. *Significantly different from $1 ; p<0.01$.

Ang II-induced nuclear translocation of STAT3. The rationale for this idea was based on our earlier observations, which showed that Ang II stimulates MAP kinase in the neurons and that this stimulation is important in the neuromodulatory actions of Ang II (Yang et al., 1996). Treatment of neuronal cells with antisense oligonucleotide (AON) to MAP kinase results in a $\sim 70-80 \%$ depletion of MAP kinase (Yang et al., 1996). Such a depletion of MAP kinase was associated with a significant attenuation of Ang II-stimulated accumulation of STAT3 into the nucleus (Fig. 3A). Sense oligonucleotide (SON) of MAP kinase showed no effect. Despite inhibition of nuclear translocation of STAT3, MAP kinase AON showed no effect on the overall phosphorylation of STAT3 in neuronal cells (Fig. $3 B$ ). These data indicate that MAP kinase is involved only in the translocation of STAT3 into the nucleus and not in its phosphorylation. This conclusion was supported by the immunofluorescence experiments presented in Figure 4. Significant staining of STAT3 was observed in the nuclei of Ang II-treated neurons (Fig. 4b). Although MAP kinase SON pretreatment showed a comparable immunoreactivity with the Ang II-treated neurons (Fig. 4d), AON-treated neurons showed
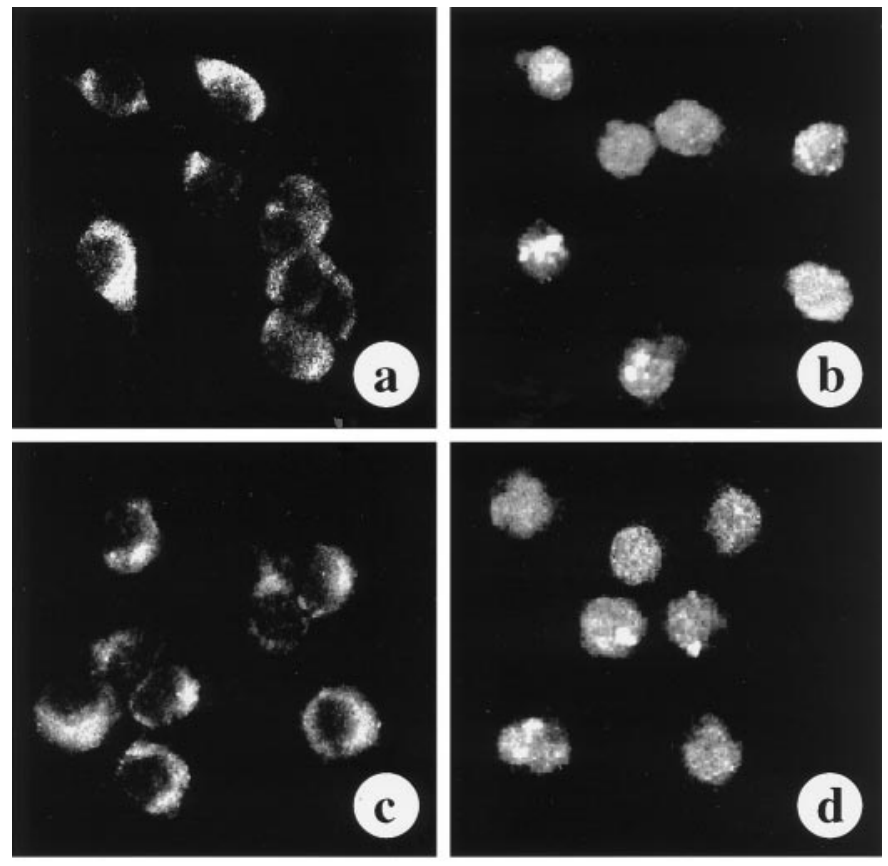

$10 \mu$

Figure 4. Effect of MAP kinase antisense oligonucleotide on immunofluorescent distribution of STAT3 in neurons. The experimental protocol was identical to that described in Figures 2 and 3. Neurons were preincubated without $(a, b)$ or with MAP kinase AON $(c)$ or with MAP kinase SON $(d)$ for $24 \mathrm{hr}$, followed by incubation without $(a)$ or with $(b$, $c, d) 100 \mathrm{~nm}$ Ang II for $30 \mathrm{~min}$.

very little STAT3 in the nucleus (Fig. 4c). Thus, MAP kinase AON blocked Ang II-stimulated transport of STAT3 into the nucleus. As a consequence STAT3 remained accumulated in the cytoplasmic compartment.

\section{Effect of Ang II on p62 phosphorylation}

Figure $5 A$ shows that Ang II stimulated, in a time-dependent manner, phosphorylation of a $\sim 62 \mathrm{kDa}$ protein that was immunoprecipitated by an anti-NPC antibody 414 . Identity of this protein was established to be the p62 on the basis of specificity of the antibody, its mobility on SDS-PAGE, and its recognition by another antibody that was specific for the p62 (Starr and Hanover, 1990). A maximal 12-fold stimulation of p62 phosphorylation was observed in $15 \mathrm{~min}$ by $100 \mathrm{~nm}$ Ang II. This stimulation was also $\mathrm{AT}_{1}$ receptor subtype-mediated because it was blocked by losartan (Fig. $5 B$ ). The role of MAP kinase on the phosphorylation of p62 was studied in view of the observations that Ang II stimulates this enzyme and that a putative MAP kinase substrate consensus sequence is present in the p62 (Cordes et al., 1991). Immunoprecipitated p62 from neuronal cells was used as a substrate for exogenous MAP kinase. Figure 6 shows that MAP kinase caused a time-dependent phosphorylation of p62. The effect of a peptide, corresponding to amino acids $189-198$ of the p62 peptide ${ }^{189-198}$ that contains the putative phosphorylation site for MAP kinase, on p62 phosphorylation further confirmed the role of MAP kinase. Peptide ${ }^{189-198}$ blocked the phosphorylation of p62 by exogenous MAP kinase in a dose-dependent manner (Fig. 7). In contrast, a mutant peptide ${ }^{189-198}$ in which $\mathrm{Thr}^{193}$ was replaced with Ala showed no effect (Fig. 7). 
A
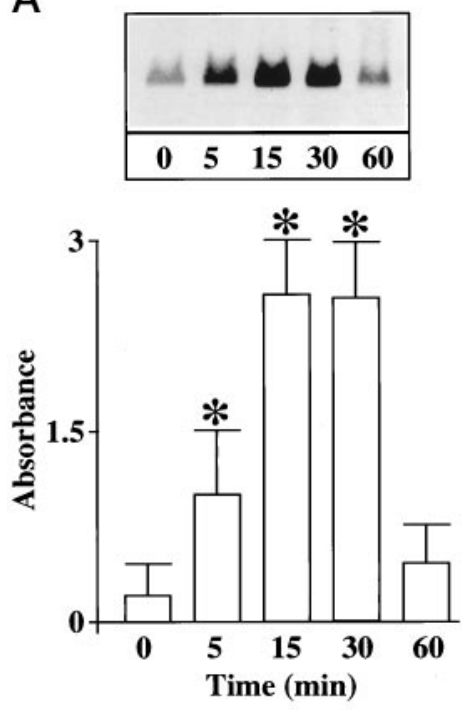

B
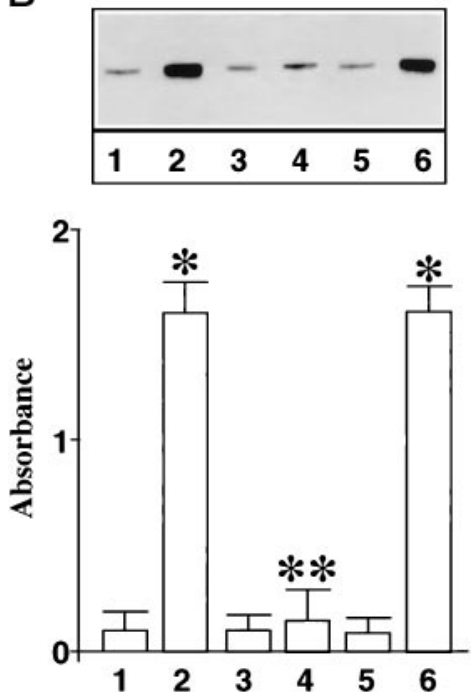

Figure 5. Ang II stimulation of p62 phosphorylation in neurons. $A$, Time course. Neuronal cultures were prelabeled with $\left[{ }^{32} \mathrm{P}\right]$-orthophosphate and incubated with $100 \mathrm{~nm}$ Ang II for $15 \mathrm{~min}$ at $37^{\circ} \mathrm{C}$; cell lysates were subjected to immunoprecipitation of p62 as described in Materials and Methods. Top, A representative autoradiogram showing a phosphoprotein of $\sim 62 \mathrm{kDa}$ corresponding to p62. Bottom, Quantitation of p62 bands from three separate experiments and data are mean \pm SE. *Significantly different from zero time $(p<0.05)$. $B$, Ang II receptor subtype specificity. Experimental protocol was identical to that described in $A$. Cultures were treated with $100 \mathrm{~nm}$ Ang II for $15 \mathrm{~min}$ in the absence or presence of Ang II receptor antagonists. 1, Control; 2, 4, 6, $100 \mathrm{~nm}$ Ang II; 3, 4, $10 \mu \mathrm{M}$ losartan; and 5, 6, $10 \mu \mathrm{M}$ PD123319. *Significantly different from $1(p<$ $0.025)$. ${ }^{* *}$ Significantly different from $2(p<0.025)$.

Neuronal cultures were pretreated with MAP kinase AON to deplete them of MAP kinase. This was followed by determining the effect on Ang II-stimulated p62 phosphorylation. MAP kinase AON treatment caused a significant blunting of Ang II stimulation of p62 phosphorylation (Fig. 8). It is the same experimental condition in which MAP kinase AON also blocks the effect of Ang II on STAT3 transport into the nucleus (Fig. 3). Treatment with MAP kinase SON showed no attenuation of the effect of Ang II. Next, neuronal cells were subjected to osmotic loading to introduce the peptide ${ }^{189-198}$

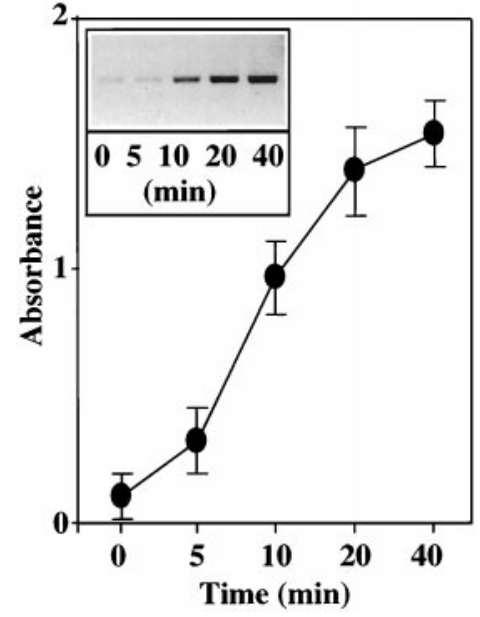

Figure 6. In vitro phosphorylation of $\mathrm{p} 62$ by MAP kinase. Neuronal cells were lysed, and p62 was immunoprecipitated. Immunoprecipitates were used as substrate for exogenous MAP kinase as described in Materials and Methods. Phosphorylated p62 bands were quantitated by SW5000 Gel Analysis System. Top, A representative autoradiogram. Bottom, Data from three separate experiments are presented as mean $\pm \mathrm{SE}$.
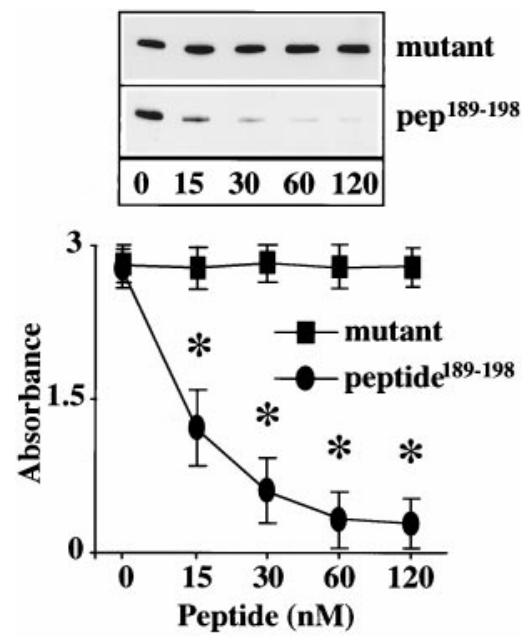

Figure 7. Inhibition of MAP kinase-mediated phosphorylation of p62 by p62 peptide ${ }^{189-198}$. Peptide ${ }^{189-198}$ (GSPFTPATLA) corresponding to amino acids 189 to 198 of p62 containing a punitive substrate consensus for MAP kinase (PXTP) was incubated with the immunoprecipitates of p62 from neurons at the indicated concentrations. In control incubations, mutant p62 peptide ${ }^{189-198}$ in which $\mathrm{Thr}^{193}$ was replaced with Ala was used under identical conditions. MAP kinase assay and p62 phosphorylation were performed as described in the legend to Figure 6. Top, Representative autoradiogram. Bottom, Mean \pm SE $(n=3)$. *Significantly different from zero time $(p<0.025)$.

into the neurons to provide an in vivo evidence for the involvement of MAP kinase in Ang II-induced p62 phosphorylation. Treatment of neurons with peptide ${ }^{189-198}$ resulted in a $85 \%$ decrease in the ability of Ang II to stimulate phosphorylation of endogenous p62 (Fig. 9). This inhibition was specific because the mutant peptide showed no effect. These data suggest that Ang II stimulates p62 phosphorylation and this phosphorylation involves MAP kinase.

\section{Role of p62 phosphorylation in nuclear translocation of STAT3}

Neuronal cells were subjected to osmotic loading protocol to introduce the peptide ${ }^{189-198}$ or its mutant counterpart under the 


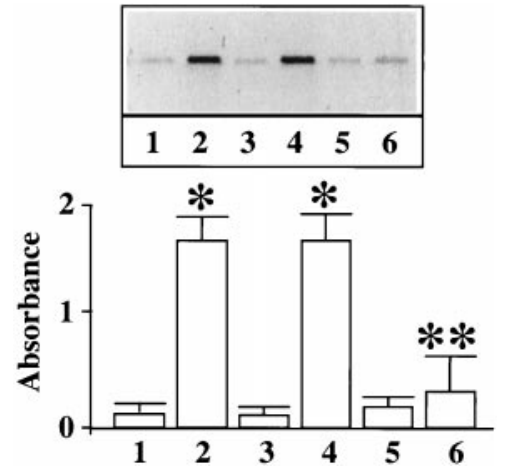

Figure 8. Effect of MAP kinase antisense oligonucleotide on Ang IIstimulated phosphorylation of p62 in neurons. Neuronal cultures were preincubated without $(1,2)$ or with $1 \mu \mathrm{M}$ MAP kinase AON $(5,6)$ or with $1 \mu \mathrm{M}$ MAP kinase SON $(3,4)$ for $24 \mathrm{hr}$ at $37^{\circ} \mathrm{C}$ to deplete MAP kinase (Yang et al., 1996). This treatment causes a $70-80 \%$ decrease in MAP kinase. Neurons were incubated without $(1,3,5)$ or with $100 \mathrm{~nm}$ Ang II $(2,4,6)$ for 15 min. p62 was immunoprecipitated and analyzed by SDS-PAGE as described in Materials and Methods. Top, A representative autoradiogram. Bottom, The band corresponding to phosphorylated p62 was quantitated, and data from three experiments are presented as mean \pm SE. *Significantly different from $1 ;(p<0.005)$. ${ }^{* *}$ Significantly different from $2 ; p<0.01$.
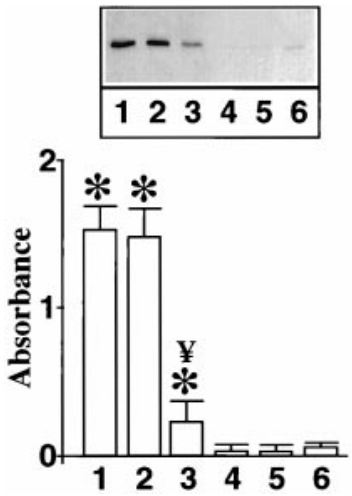

Figure 9. Effect of intracellular loading of p62 peptide ${ }^{189-198}$ on Ang II-induced phosphorylation of p62. Osmotic loading of neuronal cells without $(1,4)$ or with p62 peptide ${ }^{189-198}(3,6)$ or with its mutant $(2,5)$ was performed essentially as described in Materials and Methods. This was followed by incubation without (4-6) or with $100 \mathrm{~nm}$ Ang II (1-3) for $15 \mathrm{~min}$. Levels of phosphorylated p62 were quantitated as described in the legend to Figure 3. Top, A representative autoradiogram of phosphorylated p62. Bottom, Quantitations of the bands corresponding to p62 from three separate experiments are presented as mean \pm SE. *Significantly different from $4 ; p<0.05 ;{ }^{\ngtr}$ Significantly different from 1 and $2 ; p<0.05$.

above described condition, which results in $\sim 85 \%$ decrease in Ang II stimulation of p62 phosphorylation. This inhibition of p62 phosphorylation by the peptide ${ }^{189-198}$ was associated with a $70 \%$ decrease in STAT3 levels in the nucleus of neurons stimulated by Ang II (Fig. 10). Mutant peptides showed no such effect. Finally, cytoplasmic and nuclear fractions from control and Ang IItreated neurons were isolated. They were reconstituted in various combinations to determine whether the phosphorylation occurring at the nuclear level is the key in Ang II-induced nuclear translocation of STAT3. Incubation of cytoplasmic extract from Ang II-treated cells with the nuclei of Ang II-treated neurons resulted in a high intensity of STAT3 staining in the nuclei (Fig. $11 b)$. In contrast, incubation of a cytoplasmic fraction of Ang II-treated neurons with the nuclei isolated from control neurons
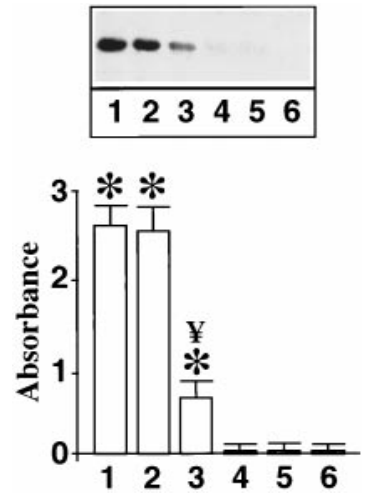

Figure 10. Effect of intracellular loading of p62 peptide ${ }^{189-198}$ on Ang II-induced nuclear translocation of STAT3. Cells were collected after loading the p62 peptide ${ }^{189-198}$ or its mutant counterpart. Phosphorylated STAT3 levels in the nuclear fraction were quantitated as described in the legend to Figure 1. Lane 1, $100 \mathrm{~nm}$ Ang II; lane 2, $100 \mathrm{~nm}$ Ang II + mutant peptide ${ }^{189-198}$; lane 3, $100 \mathrm{~nm}$ Ang II + peptide ${ }^{189-198}$; lane 4, control; lane

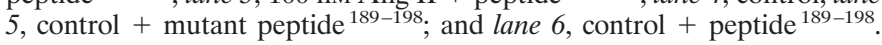
Top, A representative autoradiogram of phosphorylated STAT3. Bottom, Data from three separate experiments are mean \pm SE. *Significantly different from $4 ; p<0.005$. ${ }^{\ngtr}$ Significantly different from 1 and $2 ; p<0.05$.

showed no nuclear staining of STAT3 (Fig. 11a). The increased staining in Ang II-treated nuclei was completely attenuated when the cytoplasmic fraction of Ang II-treated neurons was incubated with the nuclear fraction from neurons that were preloaded with the peptide ${ }^{189-198}$ (Fig. 11d). However, no decrease in nuclear staining was observed when mutant peptide was used (Fig. 11c). These data indicate that Ang II treatment may have a direct effect on the p62 located on the nuclear membrane.

\section{DISCUSSION}

The observations presented in this study further our understanding regarding the signal transduction pathway involved in Ang II regulation of $\mathrm{NE}$ neuromodulation in the brain neurons. First, they establish that the $\mathrm{AT}_{1}$ receptor activation leads to the phosphorylation of p62, a glycoprotein nucleoporin that is involved in the regulation of the NPC activity. Second, Ang II stimulation of MAP kinase appears to be the protein kinase involved in this phosphorylation. Thus, phosphorylation of p62 constitutes a mechanism by which the transport activity of the NPC is accelerated. This results in an increased translocation of transcription factors and signaling molecules such as STAT3, Fos, and Jun from the cytoplasmic compartment into the nucleus where they participate in the transcriptional regulation of NE neuromodulation (Gelband et al., 1997).

Neuronal cells serve as an interesting model for studying the nuclear transport of proteins because the rate of protein trafficking is significantly higher in these cells compared with many other cells (Takahashi, 1992; Schulman, 1995). Additionally, our previous studies have shown that activation of the $\mathrm{AT}_{1}$ receptor results in an increased expression of genes relevant to $\mathrm{NE}$ synthesis and release (Lu et al., 1996a; Yu et al., 1996). This involves activation of a specific signal transduction pathway (Yang et al., 1996) followed by translocation of signaling molecules and transcription factors into the nucleus that may be key in regulating the chronic actions of Ang II (Lu et al., 1996b; Gelband et al., 1997). Coupled together, these studies suggest that the $\mathrm{AT}_{1}$ receptor stimulation in the neuron could serve as an excellent model for investigating the nuclear trafficking of signaling molecules. Finally, neurons 

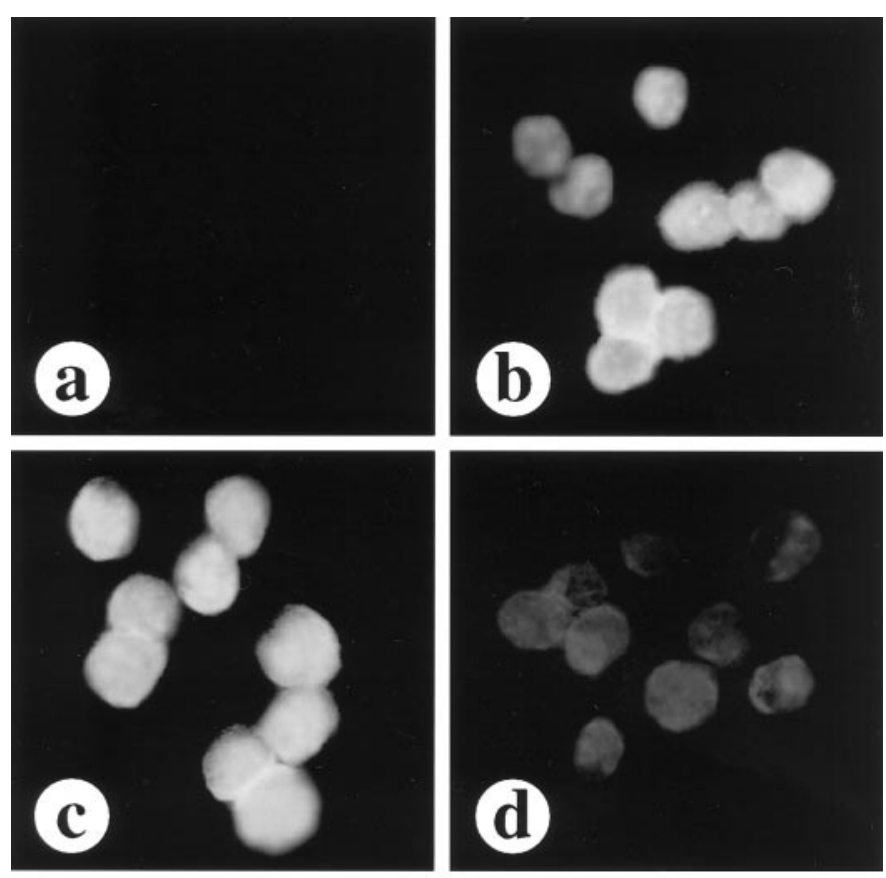

\section{$10 \mu$}

Figure 11. Effect of p62 peptide ${ }^{189-198}$ on nuclear translocation of STAT3 in isolated nuclei. Cytoplasmic fractions were prepared from neurons treated with $100 \mathrm{~nm}$ Ang II for $15 \mathrm{~min}$, as described in Materials and Methods. In addition, nuclear fractions from untreated and Ang II-treated neurons were also isolated. Cytoplasmic fraction from neurons treated with Ang II were incubated with the nuclei isolated from the neurons that are treated without $(a)$ or with $(b-d)$ Ang II in the absence $(a, b)$ or presence of p62 peptide ${ }^{189-198}(d)$ or its mutant analog $(c)$. Nuclei were fixed, and the accumulation of STAT3 in the nuclei were analyzed by anti-STAT3 antibody and confocal microscopy as described in the legend to Figure 2

provide us with the opportunity to study the role of various components of nuclear translocation system in vivo without interruption of the physiologically relevant actions of Ang II.

Our data show that the $\mathrm{AT}_{1}$ receptor stimulation results in an increased phosphorylation of STAT3, which is ultimately translocated into the nucleus. They also indicate that the phosphorylation of STAT3 is important for its nuclear translocation. In contrast, our data indicate that MAP kinase does not play any role in the phosphorylation of STAT3 in whole cells. This conclusion is based on the observation that MAP kinase-AONtreated neurons failed to exhibit inhibition of Ang II-stimulated phosphorylation of STAT3 in whole cells. Translocation of STAT3 also involves phosphorylation of p62. There are numerous lines of evidence to support this conclusion. (1) Ang II stimulates p62 phosphorylation, the characteristics of which are consistent with the activation of the $\mathrm{AT}_{1}$ receptor signaling cascade. (2) p62 phosphorylation is blocked by a peptide that competes with p62 for phosphorylation by MAP kinase both in vitro and in vivo. (3) Immunoprecipitated p62 serves as an excellent substrate for exogenous MAP kinase; (4) treatment of neurons with AON to MAP kinase attenuates Ang II stimulation of p62 phosphorylation; (5) p62 has substrate consensus sequence for MAP kinase (Cordes et al., 1991); and (6) intracellular loading of the peptide containing this consensus sequence significantly reduces Ang II stimulation of p62 phosphorylation in parallel with the inhibition of nuclear translocation of STAT3. The magnitude of inhibition of p62 phosphorylation is greater than inhibition of STAT3 translocation. This discrepancy may very well be a result of the presence of a basal rate of nuclear translocation of STAT3 that is independent of p62 phosphorylation mechanism, and (7) in vitro Ang II-induced nuclear accumulation of STAT3 was significantly attenuated by the addition of p62 peptide ${ }^{189-198}$ in the reaction mixture, suggesting that the nuclear structural component p62 is the phosphorylation site involved in the nuclear translocation of STAT3.

Finally, although our data provide evidence for the role of phosphorylation-mediated regulation of nuclear transport of macromolecules, they also raise interesting questions concerning the mechanism by which p62 phosphorylation participates in the acceleration of the transport. One could postulate that phosphorylation of p62 may regulate the docking of the "transport complex" (i.e., a complex of STAT3-NLS receptor) to the NPC, thus accelerating their translocation into the nucleus. Evidence for this hypothesis is derived from the observation showing that repetitive peptide motifs relevant to potential docking sites have been indicated in nucleoporins, including p62 (Radu et al., 1995a,b). In addition, p62 has been shown to bind to NLS receptor with high affinity, providing a basis for physical interaction between the transport complex and the NPC (Haltiwanger et al., 1992; Guan et al., 1995; Paschal and Gerace, 1995). Alternatively, because p62 is a component of the NPC, its phosphorylation could induce conformational changes in the transport channel of the NPC to regulate the capacity and speed of the transport through the channel (Akey and Radermacher, 1993). In conclusion, these data provide strong evidence for a MAP kinasedependent phosphorylation of p62 and its role in the transmission of neuromodulatory signals of Ang II from plasma membrane to the nucleus.

\section{REFERENCES}

Ahmad F, Li PM, Meyerovitch J, Goldsteiner BJ (1995) Osmotic loading of neutralizing antibody demonstrates a role for protein-tyrosine phosphatase $1 \mathrm{~B}$ in negative regulation of the insulin action pathway. J Biol Chem 270:20503-20508.

Akey CW (1992) The nuclear pore complex. Curr Opin Struct Biol $2: 258-263$.

Akey CW, Radermacher M (1993) Architecture of the Xenopus nuclear pore complex revealed by the three-dimensional cryo-electron microscopy. J Cell Biol 122:1-19.

Boulikas T (1993) Nuclear localization signals (NLS). Crit Rev Eukaryot Gene Expr 3:193-227.

Buss F, Stewart M (1995) Macromolecular interactions in the nucleoporin $\mathrm{p} 62$ complex of rat nuclear pores: binding of nucleoporin $\mathrm{p} 54$ to the rod domain of p62. J Cell Biol 128:251-261.

Cordes V, Waizenerger I, Krohne G (1991) Nuclear pore complex glycoprotein p62 of Xenopus laevis and mouse: cDNA cloning and identification of its glycosylation region. Eur J Cell Biol 55:31-47.

Davis LI (1995) The nuclear pore complex. Annu Rev Biochem 64:865-896.

Feldherr C (1995) Stimulation of nuclear import by Simon virus 40transformed cell extracts is dependent on protein kinase activity. Mol Cell Biol 15:7043-7049.

Finlay DR, Meier E, Bradley P, Horecka J, Forbes DJ (1991) A complex of nuclear pore proteins required for pore function. J Cell Biol 114:169-183.

Gelband CH, Sumners C, Raizada MK (1997) Angiotensin receptors and norepinephrine neuromodulation: implications of functional coupling. Regul Pept, in press.

Gerber UF, Gerace L (1992) Nuclear protein import is inhibited by an antibody to a lumenal epitopic of a nuclear pore complex glycoprotein. J Cell Biol 116:15-30. 
Guan T, Muller S, Klier G, Panté N, Blevitt JM, Haner M, Paschal B, Aebi U, Gerace L (1995) Structural analysis of the p62 complex, an assembly of O-linked glycoproteins that localizes near the central gated channel of the nuclear pore complex. Mol Biol Cell 6:1591-1603.

Haltiwanger RS, Kelly WG, Roquemore EP, Blomberg MA, Dong LYD, Kreppel L, Chou TY, Hart GW (1992) Glycosylation of nuclear and cytoplasmic proteins is ubiquitous and dynamic. Biochem Soc Trans 20:264-269.

Jakob R (1992) Purifying nuclei. Prep Biochem 22:1-9.

Kita K, Omata S, Horigome T (1993) Purification and characterization of a nuclear pore glycoprotein complex containing p62. J Biochem 113:377-382.

Lu D, Yang H, Raizada MK (1996a) Angiotensin II regulation of neuromodulation: downstream signaling mechanism from activation of mitogen-activated protein kinase. J Cell Biol 135:1609-1619.

Lu D, Yu K, Paddy MR, Rowland NE, Raizada MK (1996b) Regulation of norepinephrine transporter system by angiotensin II in neuronal cultures of normotensive and spontaneously hypertensive rat brains. Endocrinology 137:763-772.

Marrero MB, Schieffer B, Paxton WG, Heerdt L, Berk BC, Delafontaine P, Bernstein KE (1995) Direct stimulation of Jak/STAT pathway by the angiotensin $\mathrm{II} \mathrm{AT}_{1}$ receptor. Nature 375:247-250.

Melchior R, Gerace L (1995) Mechanisms of nuclear protein import. Curr Opin Cell Biol 7:310-318.

Melchior F, Paschal B, Evans J, Gerace L (1993) Inhibition of nuclear protein import by non-hydrolyzable analogues of GTP and identification of the small GTPase Ran/TC4 as an essential transport factor. J Cell Biol 123:1649-1659.

Miller MW, Hanover JA (1994) Functional nuclear pores reconstituted with $\beta 1-4$ galactose-modified O-link $N$-acetylglucosamine glycoproteins. J Biol Chem 269:9289-9297.

Moore MS, Blobel G (1992) The two steps of nuclear import, targeting to the nuclear envelope and translocation through the pore require different cytosolic factors. Cell 69:939-950.

Moore MS, Blobel G (1993) The GTP-binding protein Ran/TC4 is required for protein import into the nucleus. Nature 365:661-663.

Moore MS, Blobel G (1994) Purification of a Ran-interacting protein that is required for protein import into the nucleus. Proc Natl Acad Sci USA 91:10212-10216.

Newmeyer DD, Fordes DJ (1988) Nuclear import can be separated into distinct steps in vitro. Nuclear pore binding and translocation. Cell 51:641-653.

Newmeyer DD, Lucocq JM, Burglin TR, De Robertis EM (1986) Assembly in vitro of nuclei active in nuclear protein transport: ATP is required for nucleoplasmin accumulation. EMBO J 5:501-510.

Panté N, Aebi U (1994) Towards understanding the 3-D structure of the nuclear pore complex at the molecular level. Curr Opin Struct Biol 4:187-196.

Paschal BM, Gerace L (1995) Identification of $\mathrm{NTF}_{2}$, a cytosolic factor for nuclear protein import that interacts with nuclear pore complex protein p62. J Cell Biol 129:925-937.
Paxon WG, Marrero MB, Klin JD, Delafontaine P, Berk BC, Bernstein $\mathrm{KE}$ (1994) The angiotensin II $\mathrm{AT}_{1}$ receptor is tyrosine and serine phosphorylated and can serve as a substrate for the Sre family of tyrosine kinases. Biochem Biophys Res Commun 200:260-267.

Radu A, Moore MS, Blobel G (1995a) The peptide repeat domain of nucleoporin Nup98 functions as a docking site in transport across the nuclear pore complex. Cell 81:215-222.

Radu A, Blobel G, Moore MS (1995b) Identification of a protein complex that is required for nuclear protein import and mediates docking of import substrate to distinct nucleoporins. Proc Natl Acad Sci USA 92:1769-1773.

Raizada MK, Lu D, Tang W, Kurian P, Sumners C (1993) Increased angiotensin II type 1 receptor gene expression in neuronal cultures from spontaneously hypertensive rats. Endocrinology 132:1715-1722.

Raizada MK, Lu D, Sumners C (1994) $\mathrm{AT}_{1}$ receptors and angiotensin actions in the brain and neuronal cultures of normotensive and hypertensive rats. In: Current concepts: tissue renin-angiotensin system as local regulators in reproductive and endocrine organs (Mukhopadhyay AK, Raizada MK, eds) pp 331-348. New York: Plenum.

Richardson WD, Mills AD, Dilworth SM, Laskey RA, Dingwall C (1988) Nuclear protein migration involves two steps: rapid binding at the nuclear envelope followed by slower translocation through the nuclear pores. Cell 52:655-664.

Schulman H (1995) Protein phosphorylation in neuronal plasticity and gene expression. Curr Opin Neurobiol 5:375-381.

Shi Y, Thomas JO (1992) The transport of proteins into the nucleus requires the 70-kilodalton heat shock protein or its cytosolic cognate. Mol Cell Biol 12:2186-2192.

Starr CM, Hanover JA (1990) Glycosylation of nuclear pore protein p62: reticulocyte lysate catalyses $\mathrm{O}$-linked $\mathrm{N}$-acetylglucoamine addition in vitro. J Biol Chem 265:6868-6873.

Sukegawa J, Blobel G (1993) A nuclear pore complex protein that contains zinc finger motifs, binds DNA and faces the nucleoplasm. Cell 72:29-38.

Sumners C, Tang W, Zelezna B, Raizada MK (1991) Angiotensin II receptor subtypes are coupled with distinct signal transduction mechanisms in neurons and astroglia from rat brain. Proc Natl Acad Sci USA 55:7567-7571.

Takahashi Y (1992) Gene expression in cells of the central nervous system. Prog Neurobiol 38:523-569.

Yang H, Lu D, Yu K, Raizada MK (1996) Regulation of neuromodulatory actions of angiotensin II in the brain neurons by the Rasdependent mitogen-activated protein kinase pathway. J Neurosci 16:4047-4058.

Yang H, Lu D, Vinson GP, Raizada MK (1997) Involvement of MAP kinase in angiotensin II-induced phosphorylation and intracellular targeting of neural $\mathrm{AT}_{1}$ receptor. J Neurosci 17:1660-1669.

Yu K, Lu D, Rowland NE, Raizada MK (1996) Regulation of tyrosine hydroxylase gene expression in neuronal cultures of normotensive and spontaneously hypertensive rats. Endocrinology 137:3566-3576. 